\title{
Robust Resistance Network Topology Design by Conic Optimization
}

\author{
C. $\operatorname{Roos}^{\star}$, Y. Bai ${ }^{\star \star}$ and D. Chaerani ${ }^{\star \star \star}$
}

${ }^{*}$ Faculty of Information Technology and Systems, Delft University of Technology, P.O. Box 5031, 2600 GA Delft, The Netherlands,Email: c.roos@tudelft.nl.

**Shanghai University, Shanghai, China,Email: yanqin.bai@gmail.com.

${ }^{* * *}$ Jurusan Matematika, FMIPA, Universitas Padjadjaran, Bandung, Indonesia, Email: d_chaerani@yahoo.com.

\begin{abstract}
After a brief introduction to the field of Conic Optimization we present an application to the (robust) resistor network topology design problem, where the goal is to design an electrical network containing resistors, such that the dissipation is minimal, given the external current values at the nodes of the network and assuming that the conductance values satisfy some normalizing constraint. We present a linear model for the single-current case and semidefinite models for multicurrent cases. All models are illustrated by examples.
\end{abstract}

KEYWORDS: Conic optimization, Network topology design, Conic quadratic optimization, Semidefinite optimization, Robust optimization.

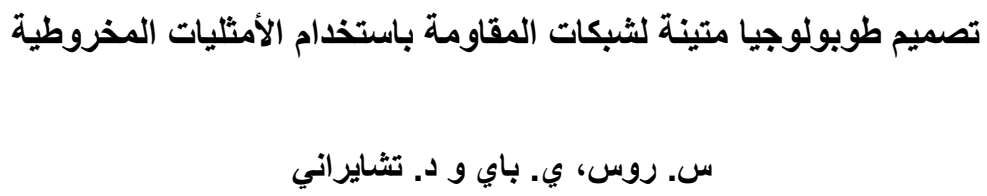

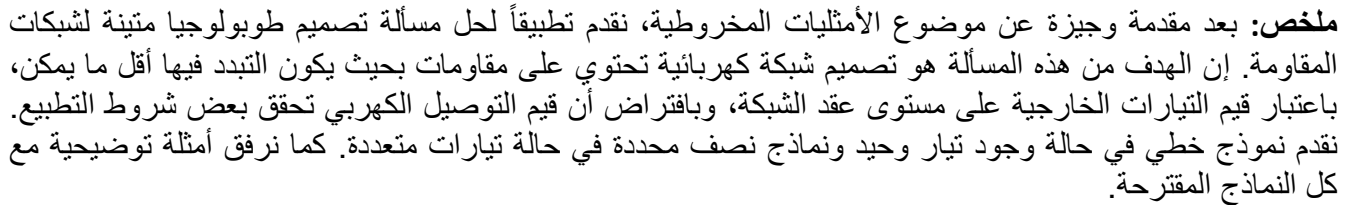

\section{Introduction}

$A$ very general mathematical setting of an optimization problem is the following:

$$
\min _{x \in X}\left\{f_{0}(x): f_{i}(x) \leq 0, i=1, \cdots, m\right\} .
$$

In this problem, we are given an objective function $f_{0}(x)$ and finitely many functional constraints $f_{i}(x) \leq 0, i=1, \cdots, m$. The functions $f_{i}(x)$ are real-valued functions of an $n$-dimensional design vector $x$ 


\section{ROOS, Y. BAI and D. CHAERANI}

varying in a given domain $X$. The goal is to minimize the objective over the feasible set of the problem, i.e., the set which is cut off the domain $X$ by the system of inequalities $f_{i}(x) \leq 0, i=1, \cdots, m$. In general, this is a very hard problem to solve. The situation is much better if the set $X$ and all functions $f_{i}(x), i=0,1, \cdots, m$ are convex. In that case $(\mathrm{P})$ is called a Convex Optimization problem. But even then, the problem might be hard to solve.

In this paper we restrict ourselves to a special class of convex optimization problems, namely Conic Optimization (CO) problems. $\mathrm{CO}$ addresses the problem of minimizing a linear objective function over the intersection of an affine set and a convex cone. The general form is as follows:

$$
\min _{x \in \mathbf{R}^{n}}\left\{c^{T} x: A x-b \in K\right\} \text {. }
$$

The objective function is $c^{T} x$, where $c \in \mathbf{R}^{n}$ is called the objective vector. Furthermore, $A x-b$ represents an affine function from $\mathbf{R}^{n}$ to $\mathbf{R}^{m}$ and $K$ denotes a convex cone in $\mathbf{R}^{m}$. Usually $A$ is given as an $m \times n$ (constraint) matrix, and $b \in \mathbf{R}^{m}$. The importance of this class of problems is due to two facts: many nonlinear problems can be modelled as a $\mathrm{CO}$ problem, and, secondly, under some weak conditions on the underlying cone $K$, CO problems can be solved efficiently.

The easiest and most well known case occurs when the cone $K$ is the nonnegative orthant of $\mathbf{R}^{m}$, i.e. when $K=\mathbf{R}_{+}^{m}$ :

$$
\min _{x \in \mathbf{R}^{n}}\left\{c^{T} x: A x-b \in \mathbf{R}_{+}^{m}\right\} .
$$

This is nothing but one of the forms of the well known Linear Optimization (LO) problem. Thus it becomes clear that LO is a special case of CO. It is well known that LO models cover numerous applications. Whenever applicable, LO allows useful quantitative and qualitative information on the problem at hand to be obtained. The specific analytic structure of an LO problem gives rise to a number of general results which in many cases provide valuable insight and understanding. At the same time, this analytic structure underlies some specific computational techniques for LO; these techniques, which by now are perfectly well developed, allow quite large LO problems (with tens/hundreds of thousands of variables and constraints) to be solved routinely. Nevertheless, in practice, many situations arise that cannot be covered by LO models. To handle these essentially nonlinear cases, there is a strong need to extend the basic theoretical results and computational techniques known for LO beyond the bounds of LO.

When passing from a generic LO problem to its nonlinear extensions, we should expect to encounter some nonlinear components in the problem. Historically, this was done by putting the nonlinearity in the functions defining the problem, as done above in problem (P). In CO, however, we replace the cone $\mathbf{R}_{+}^{m}$ in (LO) by a convex cone $K$, and hence the nonlinearity is now captured in the cone. In the next section we discuss some basic properties of relevant convex cones and we introduce two special cones that play a prominent role in the context of $\mathrm{CO}$.

In the recent years, a lot of attention has been devoted to CO. The reason is that the interior-point methods developed in the three last decades for LO, and which revolutionized the field of LO, could be naturally extended to obtain polynomial-time methods for CO (see, for example Ben-Tal and Nemirovski (2001), Nesterov and Nemirovski (1994), Roos et al. (2005), Terlaky (1973), Wright (1997) and Ye (1997)). This opened the way to a wide spectrum of new applications which cannot be captured by LO, e.g. in control theory, combinatorial optimization, etc. For a complete survey both of the theory of $\mathrm{CO}$ and its applications, we refer to the recent book by Ben-Tal and Nemirovski (2001).

The aim of this paper is to introduce the reader to the theory of $\mathrm{CO}$, and to illustrate its use. LO has a beautiful duality theory. We will see that much of this theory can be generalized to CO. In Ben-Tal and Nemirovski (2001), the authors dealt with one of the important applications of CO, namely truss topology design. We apply a similar approach to the topology design of electrical networks consisting of resistances only. 


\section{ROBUST RESISTANCE NETWORK TOPOLOGY DESIGN}

We call it the resistor network topology design (RNTD) problem. As far as we know this is a new application of topology design problems by $\mathrm{CO}$.

The paper is organized as follows. Section 2 introduces the theory of $\mathrm{CO}$ including the main duality results for CO. Section 3 is devoted to the RNTD problem. After introducing some notation in Section 3.1, we derive a nonlinear and a linear model of the single-current RNTD problem in Section 3.2 and Section 3.3, respectively. In Section 3.3 we present the solution of of a simple RNTD problem and we show that the resulting design is very unstable with respect to small perturbations of the current for which it is designed.

Based on a simple variational principle, introduced in Section 3.4.1, we derive a model for the RNTD problem that enables us to deal with the multi-current case, i.e., the case where we want to design a network that best withstands a finite set of different external currents. The model is a CO model, of the semidefinite type. It is shown that by using this model a more stable design can be obtained, but also that the new design may be still sensitive to small occasional currents. To make the design less sensitive to such perturbations in the design current, in Section 3.4.3 we make use of a recently developed modelling technique that yields a very robust design (see, for example, Ben-Tal et al. (1999, 2000), Ben-Tal and Nemirovski (1997, 1998, 1999, 2000, 2001, 2002a, 2002b), El Ghaoui and Lebret (1997), El Ghaoui et al. (1999) and Soyster (1973)). The paper concludes with some final remarks in Section 4. A preliminary version of this paper gave rise to Example 8.2.6 in Ben-Tal et al. (2009). This paper can be seen as a worked out version of this example.

\section{Conic optimization}

The general form of a $\mathrm{CO}$ problem is as given by (ConP). In this section we start with a discussion of the conditions on the cone $K$, and we review the three most important cones. Then we deal with the main duality results for $\mathrm{CO}$. It will become clear that, under some mild conditions, the duality theory for $\mathrm{CO}$ closely resembles the well known duality theory for LO.

\subsection{More on convex cones}

Recall that a subset $K$ of $\mathbf{R}^{m}$ is a cone if

$$
a \in K, \lambda \geq 0 \Rightarrow \lambda a \in K,
$$

and the cone $K$ is a convex cone if moreover

$$
a, a^{\prime} \in K \Rightarrow a+a^{\prime} \in K \text {. }
$$

We will impose three more conditions on $K$. Recall that $\mathrm{CO}$ is a generalization of LO. To obtain duality results for CO similar to those for LO, the cone $K$ should inherit three more properties from the cone underlying LO, which is the nonnegative orthant:

$$
\mathbf{R}_{+}^{m}=\left\{x=\left(x_{1} ; \cdots ; x_{m}\right): x_{i} \geq 0, i=1, \cdots, m\right\} .
$$

This cone is called the linear cone. The linear cone is not only a convex cone; it is also pointed, it is closed and it has a nonempty interior. These are exactly the three properties we need. We describe these properties now. A convex cone $K$ is called pointed if it does not contain a line. This property can be formalized as follows:

$$
a \in K,-a \in K \Rightarrow a=0 \text {. }
$$

A convex cone $K$ is called closed if it is closed under taking limits:

$$
a_{i} \in K \quad(i=1,2, \cdots), a=\lim _{i \rightarrow \infty} a_{i} \Rightarrow a \in K .
$$

Finally, denoting the interior of $K$ as int $K$, we require that

$$
\text { int } K \neq \varnothing \text {. }
$$

This means that $K$ is full-dimensional, i.e., there exists a vector $x \in K$ such that a ball of positive radius centered at $x$ is contained in $K$. In CO we only deal with cones $K$ that enjoy the three above properties. So we always assume that $K$ is a pointed and closed convex cone with nonempty interior. Apart from the linear cone, two other relevant examples of such cones are 


\section{ROOS, Y. BAI and D. CHAERANI}

1. The Lorentz cone

$$
\mathbf{L}^{m}=\left\{x \in \mathbf{R}^{m}: x_{m} \geq \sqrt{x_{1}^{2}+\cdots+x_{m-1}^{2}}\right\} .
$$

This cone is also called the second-order cone, or the ice-cream cone.

2. The positive semidefinite cone $\mathbf{S}_{+}^{m}$. This cone 'lives' in the space $\mathbf{S}^{m}$ of $m \times m$ symmetric matrices (equipped with the Frobenius inner product $\left.\langle A, B\rangle=\operatorname{Tr}(A B)=\sum_{i, j} A_{i j} B_{i j}\right)$ and consists of all $m \times m$ matrices $A$ which are positive semidefinite, i.e.,

$$
\mathbf{S}_{+}^{m}=\left\{A \in \mathbf{S}^{m}: x^{T} A x \geq 0, \quad \forall x \in \mathbf{R}^{m}\right\} .
$$

We assume that the cone $K$ in $(\mathrm{ConP})$ is a direct product of the form

$$
K=K^{1} \times \cdots \times K^{m},
$$

where each component $K^{i}$ is either a linear, a Lorentz or a semidefinite cone.

If $x \in K$ we also denote this as $x \succeq_{K} 0$, and if $x \in \operatorname{int} K$ then we write $x \succ_{K} 0$. If this gives no confusion we omit the argument $K$ in these notations. For example, if $A$ is a square matrix, then $A \succeq 0$ means that $A$ is positive semidefinite.

\subsection{Conic duality}

Before we derive the duality theory for $\mathrm{CO}$, we need to define the dual cone of a convex cone $K$ :

$$
K_{*}=\left\{\lambda \in \mathbf{R}^{m}: \lambda^{T} a \geq 0, \forall a \in K\right\} .
$$

We recall the following result from Ben-Tal and Nemirovski (2001).

Theorem 2.1 Let $K \subset \mathbf{R}^{m}$ be a nonempty cone. Then

(i) the set $K_{*}$ is a closed convex cone;

(ii) if $K$ has a nonempty interior (i.e., int $K \neq \varnothing$ ) then $K_{*}$ is pointed;

(iii) if $K$ is a closed convex pointed cone, then int $K_{*} \neq \varnothing$;

(iv) if $K$ is a closed convex cone, then so is $K_{*}$, and the cone dual to $K_{*}$ is $K$.

Corollary 2.2 If $K \subset \mathbf{R}^{m}$ is a closed pointed convex cone with nonempty interior then so is $K_{*}$, and vice versa.

If $K_{*}=K$, then $K$ is called self-dual. We leave it to the reader to verify that the three cones introduced in Section 2.1 are self-dual. Moreover, the dual of a direct product of convex cones is the direct product of their duals, i.e.,

$$
K=K^{1} \times \cdots \times K^{m} \Rightarrow K_{*}=K_{*}^{1} \times \cdots \times K_{*}^{m} .
$$

As a consequence, any direct product of linear, Lorentz and semidefinite cones is self-dual.

Now we are ready to deal with the problem dual to a conic problem (ConP). We start with observing that whenever $x$ is a feasible solution for (ConP) then the definition of $K_{*}$ implies $\lambda^{T}(A x-b) \geq 0$, for all $\lambda \in K_{*}$, and hence $x$ satisfies the scalar inequality

$$
\lambda^{T} A x \geq \lambda^{T} b, \quad \forall \lambda \in K_{*} .
$$

It follows that whenever $\lambda \in K_{*}$ satisfies the relation

$$
A^{T} \lambda=c
$$




\section{ROBUST RESISTANCE NETWORK TOPOLOGY DESIGN}

then one has

$$
c^{T} x=\left(A^{T} \lambda\right)^{T} x=\lambda^{T} A x \geq \lambda^{T} b=b^{T} \lambda
$$

for all $x$ feasible for (ConP). So, if $\lambda \in K_{*}$ satisfies (7), then the quantity $b^{T} \lambda$ is a lower bound for the optimal value of (ConP). The best lower bound obtainable in this way is the optimal value of the problem

$$
\max _{\lambda \in \mathbf{R}^{m}}\left\{b^{T} \lambda: A^{T} \lambda=c, \lambda \in K_{*}\right\} .
$$

By definition, (ConD) is the dual problem of (ConP). Using Theorem 2.1(iv), one easily verifies that the duality is symmetric: the dual problem is conic and the problem dual to the dual problem is the primal problem.

Indeed, from the construction of the dual problem it immediately follows that we have the weak duality property: if $x$ is feasible for (ConP) and $\lambda$ is feasible for (ConD), then

$$
c^{T} x-b^{T} \lambda \geq 0 \text {. }
$$

The crucial question is, of course, whether we have equality of the optimal values whenever (ConP) and (ConD) have optimal values. Differently from the LO case, however, this holds only if some additional conditions are satisfied. The following theorem clarifies the situation. For its proof we refer again to Ben-Tal and Nemirovski (2001). We call the problem (ConP) solvable if it has a (finite) optimal value, and this value is attained. Before stating the theorem it may be worth pointing out that a finite optimal value is not necessarily attained. For example, the semidefinite problem

$$
\min _{x, y \in \mathbf{R}}\left\{x:\left[\begin{array}{ll}
x & 1 \\
1 & y
\end{array}\right] \succeq 0\right\}
$$

has optimal value 0 , but one may easily verify that this value is not attained. We need one more definition: if there exists an $x$ such that $A x-b \in$ int $K$, then we say that (ConP) is strictly feasible. Thus we have similar, and obvious, definitions for (ConD) being solvable and strictly feasible, respectively.

Theorem 2.3 Let the primal problem (ConP) and its dual problem (ConD) be as given above. Then one has

i. (a) If (ConP) is below bounded and strictly feasible, then (ConD) is solvable and the respective optimal values are equal.

(b) If (ConD) is above bounded and strictly feasible, then (ConP) is solvable, and the respective optimal values are equal.

ii. Suppose that at least one of the two problems (ConP) and (ConD) is bounded and strictly feasible. Then a primal-dual feasible pair $(x, \lambda)$ is comprised of optimal solutions to the respective problems

(a) if and only if $b^{T} \lambda=c^{T} x \quad$ (zero duality gap) and

(b) if and only if $\lambda^{T}[A x-b]=0 \quad$ (complementary slackness).

Note that this result is slightly weaker than the corresponding result for the LO case. In the LO case the same theorem holds by putting everywhere 'feasible' instead of 'strictly feasible'. However, the adjective 'strictly' cannot be omitted here. For a more extensive discussion and some appropriate counter-examples we refer to Ben-Tal and Nemirovski (2001).

\section{The resistance network topology design problem}

A resistance network is an electrical network comprised of resistors only. The resistors are linked to each other; the points at which they are linked to each other are called the nodes of the network. We consider the situation where the input and output currents at the nodes are given. Our aim is to design a resistance network that minimizes the dissipation, i.e., the energy consumption, of the network. We call this the Resistance Network Topology Design Problem (RNTD) problem). 


\section{ROOS, Y. BAI and D. CHAERANI}

\subsection{Preliminaries}

We assume that some of the nodes are connected to earth. These nodes are called fixed nodes; the remaining nodes are called free nodes. Let $\bar{V}(V)$ denote the set of (free) nodes in the network. For each node $v \in \bar{V}, \quad \bar{y}_{v}$ will denote the potential of $v$. If $v$ is a fixed (i.e., grounded) node then $\overline{y_{v}}=0$, so $\bar{y}_{v}$ can be nonzero only if $v$ is a free node.

To each resistor we associate a directed arc $(v, w)$ connecting the nodes of the resistor. The set of all arcs is denoted as $A$. The node-arc incidence matrix of the resulting directed network is denoted as $\bar{B}$. So $\bar{B}$ is a $|\bar{V}| \times|A|$ matrix. If $u \in \bar{V}$ and $a=(v, w) \in A$ then $\bar{B}(u, a)=1$ if $u=v, \bar{B}(u, a)=-1$ if $u=w$, and $\bar{B}(u, a)=0$ otherwise. In other words,

$$
\bar{B}(u,(v, w))=\left\{\begin{aligned}
1, & \text { if } u=v, \\
-1, & \text { if } u=w, \quad u \in \bar{V}, \quad(v, w) \in A . \\
0, & \text { otherwise. }
\end{aligned}\right.
$$

The matrix obtained by removing the rows of $\bar{B}$ corresponding to fixed nodes is called the structural matrix of the network and denoted as $B$. In Belevitch (1968), matrix $B$ is called the partition matrix of the network.

The vector of external currents is denoted as a vector $f \in \mathbf{R}^{V}$. So $f(v)$ denotes the external current in the free node $v ; f(v)$ may be either positive, negative or zero. The currents on the arcs in the network are denoted as $x_{v w}$, and $x$ is the vector of all currents. So $x \in \mathbf{R}^{A}$. Kirchhoff's first law gives the balance equations in the free nodes, which are simply

$$
B x=f .
$$

We assume that for every free node $v$ such that $f(v) \neq 0$ there exists some path in the network to a fixed node. Then (9) certainly has a solution.

We denote by $\bar{y}(y)$ the vector of the potentials at the (free) nodes. So $\bar{y}_{v}=0$ if $v$ is fixed, and otherwise $\bar{y}_{v}=y_{v}$. By Ohm's law, on each arc $a=(v, w) \in A$ we must have $x_{v w} r_{v w}=\bar{y}_{v}-\bar{y}_{w}$, where $r_{v w}$ denotes the resistance value of the arc. In other words, since $\bar{y}_{v}-\bar{y}_{w}=\bar{b}_{v w}^{T} \bar{y}$, where $\bar{b}_{v w}$ denotes the column of $\bar{B}$ corresponding to $\operatorname{arc}(v, w)$,

$$
\bar{B}^{T} \bar{y}=R x,
$$

where $R$ denotes the diagonal matrix of size $|A| \times|A|$ with the resistance values on the diagonal, i.e. $R=\operatorname{diag}(r)$. Since $\bar{y}_{v}=0$ for all fixed nodes, we have $\bar{B}^{T} \bar{y}=B^{T} y$. Thus we obtain

$$
B^{T} y=R x \text {. }
$$

Note that if $r_{v w}=0$, then we may identify the nodes $v$ and $w$. Thus we may assume without loss of generality that $r_{v w}>0$ for all $\operatorname{arcs}(v, w)$. We allow resistance values to be $\infty$ (if the corresponding nodes are not connected). The conductance value of $\operatorname{arc}(v, w)$ is denoted as $g_{v w}$. So, letting $1 / \infty=0, g_{v w}=1 / r_{v w}$, for each arc. Defining $G=\operatorname{diag}(g)$, where $g$ is the vector of conductance values, we have $G=R^{-1}$, and equation (10) is equivalent to

$$
G B^{T} y=x
$$

Substitution into (9) yields

$$
B G B^{T} y=f .
$$




\section{ROBUST RESISTANCE NETWORK TOPOLOGY DESIGN}

The matrix $B G B^{T}$ is called the conductance matrix of the network. This is a $|V| \times|V|$ matrix. Note that the conductance matrix depends linearly on the conductance values. Without loss of generality we assume that the conductance matrix is nonsingular. From a physical point of view, it is obvious that whenever (12) has a solution then this solution is unique. In fact, one may prove that the matrix $B G B^{T}$ is positive definite if and only if the (undirected) subgraph consisting of all edges with positive conductance values is connected (Gosh et al., 2008).

To complete our model, we note that the dissipation of the network is a function of the external current vector $f$ and the conductance vector $g$. We denote it as $\operatorname{Diss}_{f}(g)$. One has

$$
\operatorname{Diss}_{f}(g)=\sum_{(v, w) \in A} x_{v w}^{2} R_{v w}=x^{T} R x \text {. }
$$

Using (10), (9) and (12), respectively, this can be reduced as follows:

$$
\operatorname{Diss}_{f}(g)=x^{T} R x=x^{T}\left(B^{T} y\right)=(B x)^{T} y=f^{T} y .
$$

Purely to illustrate the use of the above relations, we include some elementary examples.

\section{Example 3.1 (Replacement resistance of a ladder network)}

With the tools developed so far we can easily derive both well known and new formulae for replacement resistances. Obviously, when entering a unit current $e_{v}$ at a free node $v$, the replacement resistance from node $v$ to earth is equal to the potential $y_{v}$ at the same node. Due to (12), we conclude that the replacement resistance at any free node is equal to the corresponding element on the diagonal of the inverse matrix of the conductance matrix $B G B^{T}$. As an example we consider the network drawn in Figure 1. The nodes are numbered as indicated and the resistance values at the drawn arcs all are equal to 1 . The nodes 5 to 9 are taken to be fixed. The nonzero elements in the matrix $\bar{B}$ are as follow below. (In this paper, we orient the arcs such that the node numbers are increasing along the arcs, but any other choice is admissible.)

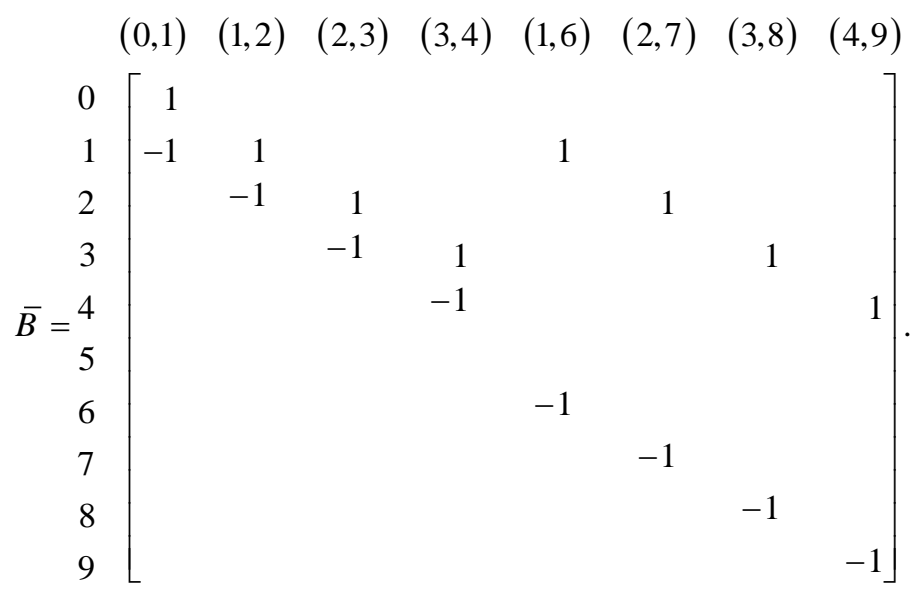

Removing the rows corresponding to fixed nodes we get the structural matrix $B$ : 


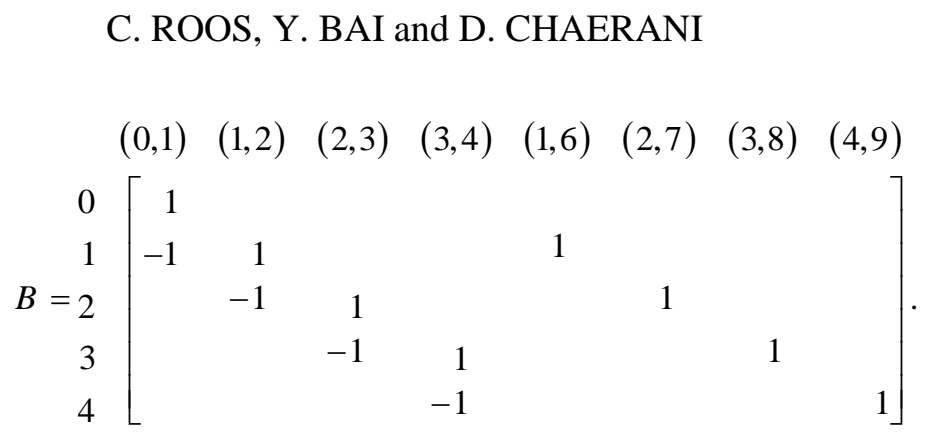

Since $R$ is the unit matrix $I$, we have $G=I$ and hence the conductance matrix satisfies $B G B^{T}=B B^{T}$ :

$$
B G B^{T}=\left[\begin{array}{rrrrr}
1 & -1 & & & \\
-1 & 3 & -1 & & \\
& -1 & 3 & -1 & \\
& & -1 & 3 & -1 \\
& & & -1 & 2
\end{array}\right] \text {. }
$$

The inverse matrix is

$$
\left(B G B^{T}\right)^{-1}=\frac{1}{21}\left[\begin{array}{rrrrr}
34 & 13 & 5 & 2 & 1 \\
13 & 13 & 5 & 2 & 1 \\
5 & 5 & 10 & 4 & 2 \\
2 & 2 & 4 & 10 & 5 \\
1 & 1 & 2 & 5 & 13
\end{array}\right] .
$$

If we want to know the resistance matrix at node 0 , we just take $f=[1 ; 0 ; 0 ; 0 ; 0]$, which gives $y=[34 ; 13 ; 5 ; 2 ; 1] / 21$, whence $y_{1}=34 / 21$. We conclude, that the replacement resistance at node 0 , i.e., the resistance from node 0 to earth, equals 34/21. Similarly, at nodes 1 and 4 the replacement resistance is 13/21, and at nodes 2 and 3 the replacement resistance is 10/21.

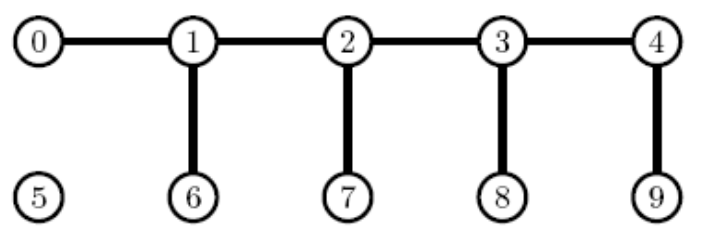

Figure 1. Ladder network of length 5.

In the above example we dealt with a ladder network of length 5 . We can easily compute the replacement resistance $r_{n}$ of the ladder network of length $n(n \geq 2)$ by using the recursive formula

$$
r_{n}=1+\frac{r_{n-1}}{1+r_{n-1}}, \quad r_{2}=2
$$

From this one easily deduces that $r_{5}=34 / 21$, as it should, and also that $r_{n}$ converges to $(1+\sqrt{5}) / 2$ if $n$ goes to infinity. 


\section{ROBUST RESISTANCE NETWORK TOPOLOGY DESIGN}

\section{Example 3.2 (Resistance between two adjacent points in a rectangular grid)}

An old question is to find the resistance between two adjacent grid points of an infinite square lattice in which all the edges represent identical resistances $R$. The result is $R / 2$, and an elegant and elementary solution of the problem is given by Aitchison (1964). We consider a grid of size $m \times n$ with $m$ odd and $n$ even. For $m=3$ and $n=4$ the network is depicted in Figure 2. We assume $R=1$. We want to know the resistance between nodes 6 and 7; these are the end nodes of the resistance in the heart of the network. This resistance is equal to the potential at node 6 when connecting node 7 to earth and entering a unit current at node 6 .

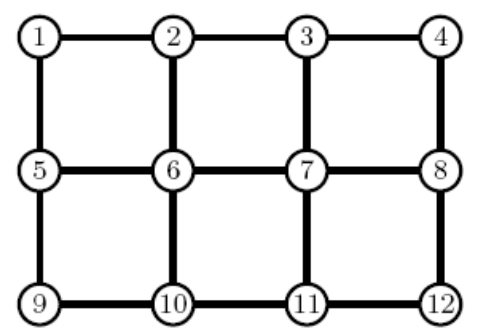

Figure 2. Example of a grid network.

As in the previous example, we use a value which is precisely the value of the element on the diagonal of the inverse matrix of the conductance matrix $B G B^{T}$ corresponding to element 6 . Table 1 gives the results for some small values of $m$ and $n$ :

Table 1. Replacement resistance of 'central' arc in a grid network

\begin{tabular}{|c|cccccc|}
\hline$m \backslash n$ & 2 & 4 & 6 & 8 & 10 & 12 \\
\hline 1 & 1.000000 & 1.000000 & 1.000000 & 1.000000 & 1.000000 & 1.000000 \\
3 & 0.600000 & 0.565217 & 0.563636 & 0.563567 & 0.563564 & 0.563564 \\
5 & 0.578947 & 0.526882 & 0.522166 & 0.521701 & 0.521652 & 0.521647 \\
7 & 0.577465 & 0.519923 & 0.512364 & 0.511120 & 0.510897 & 0.510856 \\
9 & 0.577358 & 0.518430 & 0.509238 & 0.507202 & 0.506696 & 0.506566 \\
11 & 0.577351 & 0.518098 & 0.508154 & 0.505538 & 0.504735 & 0.504477 \\
\hline
\end{tabular}

Table 2. Replacement resistance of 'central' arc when $k$ increases

\begin{tabular}{|c|ccc|}
\hline$k$ & $m$ & $n$ & replacement resistance \\
\hline 1 & 1 & 2 & 1.0000000000000000 \\
2 & 3 & 4 & 0.5652173913043479 \\
3 & 5 & 6 & 0.5221664118803231 \\
4 & 7 & 8 & 0.5111196478156734 \\
5 & 9 & 10 & 0.5066959790643568 \\
6 & 11 & 12 & 0.5044767482078642 \\
7 & 13 & 14 & 0.5032046931553875 \\
8 & 15 & 16 & 0.5024076601669391 \\
9 & 17 & 18 & 0.5018752216213316 \\
10 & 19 & 20 & 0.5015018885720043 \\
\hline
\end{tabular}

By taking $m=2 k-1$, and $n=2 k$ we found the values in Table 2 for $k=1, \cdots, 10$. If $k$ goes to infinity the value converges to 0.5 , in agreement with the aforementioned result of Aitchison (1964). 


\section{ROOS, Y. BAI and D. CHAERANI}

\subsection{Nonlinear model for the RNTD problem}

With the results of the previous section we can now build a mathematical model for the RNTD problem. We assume that the nodes are given and also which of these nodes are fixed. Furthermore, the external current $f$ is given. We want to find conductance values in such a way that the dissipation is minimized. Mathematically speaking, this amounts to solving the following minimization problem in the vector $y$ of the potentials and the vector $g$ of conductance values:

$$
\min _{g, y}\left\{f^{T} y: B G B^{T} y=f, g \geq 0\right\},
$$

where $G=\operatorname{diag}(g)$. If $y$ and $g$ are feasible, and $\lambda>0$, then $g / \lambda$ and $\lambda y$ are feasible as well, whereas the objective value is multiplied by $\lambda$. Hence, letting $\lambda$ decrease to zero, the objective value approaches to zero, but then all conductance values go to infinity, which means that all resistance values go to zero.

To prevent this pathological situation, we add a normalizing constraint which requires that the sum of the conductances is bounded above by some positive constant $\omega$. We may think of the number $\omega$ as a measure for the amount of copper that is available for connecting the nodes. The more the amount of available copper the larger the sum of the conductance values can be and the smaller the dissipation in the network. Thus we arrive at the following model:

$$
\min _{g, y}\left\{f^{T} y: B G B^{T} y=f, g \geq 0, \sum_{(v, w) \in A} g_{v w} \leq \omega\right\} .
$$

Below we use the shorthand notation $A(g)$ for the conductance matrix $B G B^{T}$. Thus we reformulate the above problem as

$$
\min _{g, y}\left\{f^{T} y: A(g) y=f, e^{T} g \leq \omega, g \geq 0\right\},
$$

where $e$ denotes the all-in-one vector in $\mathbf{R}^{A}$.

Note that as a consequence of the above argument the constraint $e^{T} g \leq \omega$ will always be tight at an optimal solution. Moreover, if $g *$ and $y *$ are optimal solutions for $\omega=1$, then for other values of $\omega$ the optimal solutions are $\omega g *$ and $y * / \omega$. Also, since $A(g)$ is linear in $g$, the equality constraints in (14) are nonlinear (they are in fact bilinear in $g$ and $y$ ). As a consequence, from a practical point of view, the model (14) cannot be solved efficiently. In the next section we present a much simpler, linear and hence computationally tractable, model.

\section{Example 3.3}

Consider a network with 4 nodes, numbered from 1 to 4 . We take node 1 to be fixed and the external currents in the nodes 2,3 and 4 to be $4,-8$ and -16 respectively. Thus we have

$$
\begin{aligned}
& (1,2) \quad(1,3) \quad(1,4) \quad(2,3) \quad(2,4) \quad(3,4) \\
& B=3 \begin{array}{r}
2 \\
4
\end{array}\left[\begin{array}{lllrcr}
-1 & & & 1 & 1 & \\
& -1 & & -1 & & 1 \\
& & -1 & & -1 & -1
\end{array}\right], \quad f=\left[\begin{array}{c}
4 \\
-8 \\
-16
\end{array}\right] .
\end{aligned}
$$

With $\omega=6$, we computed the dissipation for two different values of $g$. The results were as depicted in Figure 3. If $g_{v w}=0$, then $\operatorname{arc}(v, w)$ is not drawn. The numbers next to the arcs show the entries of the current vector $x$, and those next to the nodes the entries of the potential vector $y$. The dissipations are 184 and 96 , respectively. As will become clear later on, the last network is an optimal solution of the nonlinear problem (14). 


\section{ROBUST RESISTANCE NETWORK TOPOLOGY DESIGN}

\subsection{Linear model for the RNTD problem}

The voltage over resistor $r_{v w}$ is given by

$$
\pi_{v w}=\left|y_{v}-y_{w}\right|=\left|y^{T} b_{v w}\right|,
$$

where $b_{v w}$ denotes the column of $B$ corresponding to $\operatorname{arc}(v, w)$. In practice, it is desired that the voltages do not exceed a threshold value. Therefore, it becomes natural to impose an upper bound on the voltage values. Taking

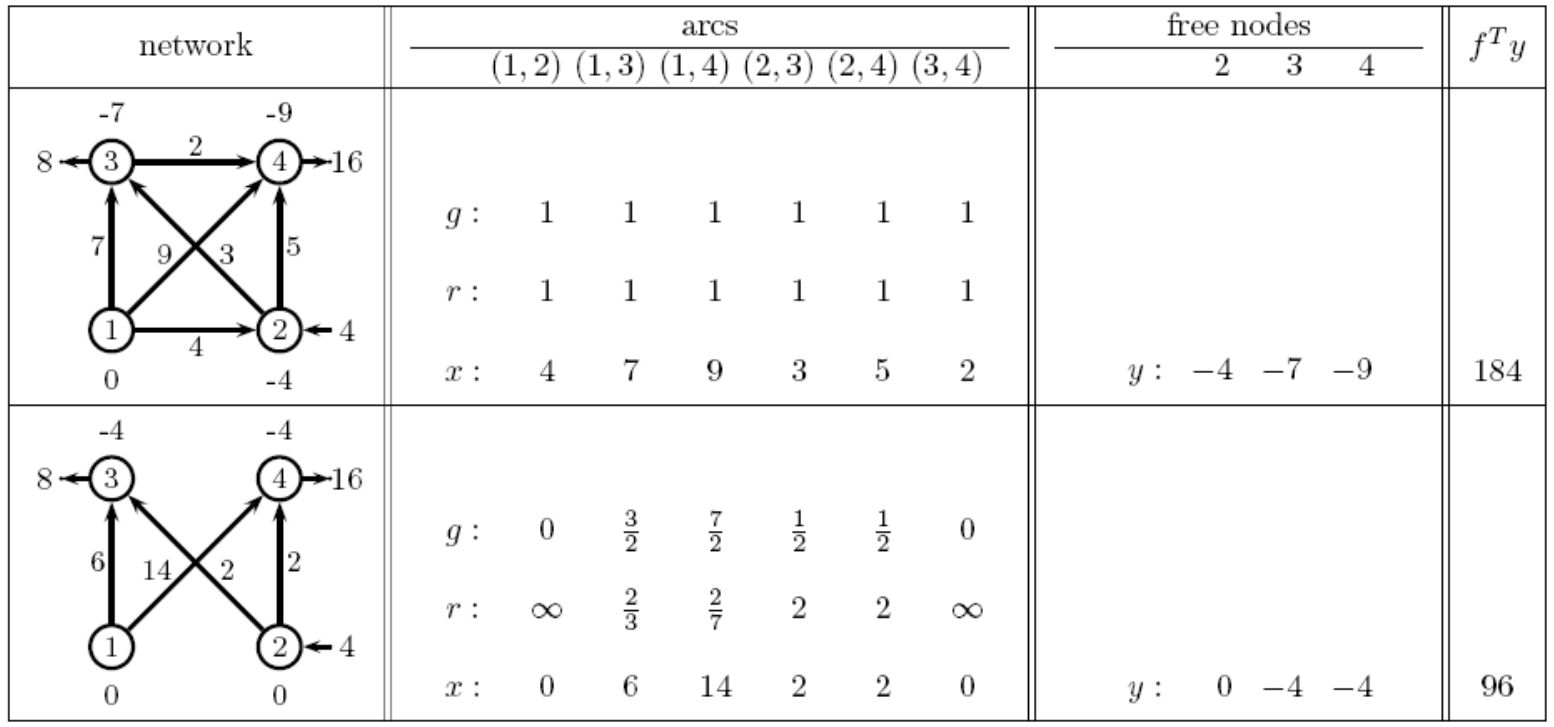

Figure 3. Several 4-node networks for $\omega=6$ and $f=(0 ; 4 ;-16 ;-4)$.

this upper bound to be equal to 1, we therefore consider the following maximization problem:

$$
\max _{y}\left\{f^{T} y:\left|y^{T} b_{v w}\right| \leq 1, \forall(v, w) \in A\right\} .
$$

In this section we deal with the surprising fact that any optimal solution of the linear problem (15) and its dual problem can be used to construct an optimal solution to the nonlinear model (14). The nonlinear constraint $\left|y^{T} b_{v w}\right| \leq 1$ can be replaced by $-1 \leq y^{T} b_{v w} \leq 1$, which makes the problem a linear optimization problem. Also note that the constrains in (15) can be written as $\left\|B^{T} y\right\|_{\infty} \leq 1$. The dual problem of (15) is given by

$$
\min _{x}\left\{\|x\|_{1}=\sum_{(v, w) \in A}\left|x_{v w}\right|: \sum_{(v, w) \in A} x_{v w} b_{v w}=f\right\} .
$$

The constraint in the dual problem can be written simply as $B x=f$. Since this equation has a solution, the dual problem is feasible. The primal problem is feasible as well (take $y=0$ ). Hence, by the duality theorem for linear optimization, both problems have optimal solutions and their optimal values are equal. If $y$ is primal feasible and $x$ dual feasible, then we may write

$$
f^{T} y=\sum_{(v, w) \in A} x_{v w} y^{T} b_{v w} \leq \sum_{(v, w) \in A}\left|x_{v w}\right|\left|y^{T} b_{v w}\right| \leq \sum_{(v, w) \in A}\left|x_{v w}\right|=\|x\|_{1},
$$




\section{ROOS, Y. BAI and D. CHAERANI}

which is nothing else than the weak duality property in the current situation. Hence, $y$ and $x$ are optimal, for (15) and (16) respectively, if and only if the above two inequalities hold with equality, and this holds if and only if

$$
x_{v w} y^{T} b_{v w}=\left|x_{v w}\right|, \quad \forall(v, w) \in A .
$$

It is worth pointing out an important consequence of this result, namely that if $y$ is primal feasible and $x$ dual feasible, then these solutions are optimal for (15) and (16) if and only if:

$$
\text { for every } \operatorname{arc}(v, w) \text { with nonzero current the voltage }\left|y^{T} b_{v w}\right| \text { equals } 1 .
$$

Also note that (17) is equivalent to

$$
\operatorname{diag}(x) B^{T} y=|x|
$$

\subsubsection{Correctness of the linear model}

In this section we show that if $y^{*}$ and $x^{*}$ satisfy (17) (i.e., are optimal for (15) and (16) respectively) then

$$
\bar{g}:=\omega \frac{\left|x^{*}\right|}{\left\|x^{*}\right\|_{1}}, \quad \bar{y}:=\frac{\left\|x^{*}\right\|_{1}}{\omega} y^{*}
$$

is an optimal solution to the nonlinear model (14). We first show that these $\bar{g}$ and $\bar{y}$ are feasible for (14), and after this that they are also optimal.

One obviously has $\bar{g} \geq 0$. The inequality $e^{T} \bar{g} \leq \omega$ holds with equality, because we may write

$$
e^{T} \bar{g}=\omega \frac{e^{T}\left|x^{*}\right|}{\left\|x^{*}\right\|_{1}}=\omega
$$

showing that the normalizing constraint is tight. Furthermore,

$$
A(\bar{g}) \bar{y}=B \operatorname{diag}(\bar{g}) B^{T} \bar{y} \stackrel{(20)}{=} B \operatorname{diag}\left(\left|x^{*}\right|\right) B^{T} y^{*} \stackrel{(19)}{=} B^{T} x^{*} \stackrel{(16)}{=} f .
$$

Thus we have shown that $\bar{g}$ and $\bar{y}$ are feasible for (14). It remains for us to show that the objective value $f^{T} \bar{y}$ is minimal. We start by computing this value for the solution given by (20). One has

$$
f^{T} \bar{y} \stackrel{(16)}{=} \bar{y}^{T} B x^{*} \stackrel{(20)}{=} \frac{\left\|x^{*}\right\|_{1}}{\omega} y * *^{T} B x^{*}=\frac{\left\|x^{*}\right\|_{1}}{\omega} e^{T}\left(\operatorname{diag}\left(x^{*}\right) B^{T} y^{*}\right) \stackrel{(19)}{=} \frac{\left\|x^{*}\right\|_{1}}{\omega} e^{T}\left|x^{*}\right|=\frac{\left\|x^{*}\right\|_{1}^{2}}{\omega} \text {. }
$$

Thus we must further show that if $g$ and $y$ are arbitrary feasible solutions to (14), then

$$
f^{T} y \geq \frac{\left\|x^{*}\right\|_{1}^{2}}{\omega}
$$

where $x^{*}$ is any optimal solution of (16). To show this we define, according to (11),

$$
x:=G B^{T} y \text {. }
$$

Then, since $g$ and $y$ are feasible solutions of (14), we have

$$
B x=B G B^{T} y=A(g) y=f,
$$

demonstrating that $x$ is feasible for (16). Since $x^{*}$ is optimal for (16), we conclude that

$$
\|x\|_{1} \geq\|x *\|_{1} \text {. }
$$

The last step in this analysis consists of proving that (23) implies (22). We have

$$
f^{T} y=(B x)^{T} y=\left(B G B^{T} y\right)^{T} y=y^{T} B G B^{T} y=\left(G B^{T} y\right)^{T} R\left(G B^{T} y\right)=x^{T} R x=\left\|R^{\frac{1}{2}} x\right\|^{2} .
$$

On the other hand, using $e=R g=R^{\frac{1}{2}} g^{\frac{1}{2}}$ and the Cauchy-Schwarz inequality we obtain 


\section{ROBUST RESISTANCE NETWORK TOPOLOGY DESIGN}

$$
\|x\|_{1}^{2}=\left(e^{T}|x|\right)^{2}=\left[\left(g^{\frac{1}{2}}\right)^{T}\left(R^{\frac{1}{2}}|x|\right)\right]^{2} \leq\left\|g^{\frac{1}{2}}\right\|^{2}\left\|R^{\frac{1}{2}} x\right\|^{2}=\left(e^{T} g\right)\left\|R^{\frac{1}{2}} x\right\|^{2} \leq \omega f^{T} y,
$$

where the last equality follows since $e^{T} g \leq \omega$ and $f^{T} y=\left\|R^{\frac{1}{2}} x\right\|^{2}$. Hence, also using $\omega>0$ and (23), we obtain

proving (22).

$$
f^{T} y \geq \frac{\|x\|_{1}^{2}}{\omega} \geq \frac{\|x *\|_{1}^{2}}{\omega}
$$

Thus we have shown that optimal solutions of the linear problem (15) and its dual (16) contain all the information we need to obtain an optimal solution of the nonlinear problem (14). The above proof makes clear that in any optimal solution we have $\|x\|_{1}^{2}=\omega f^{T} y$, and due to (24) this holds if and only if

$$
\left[\left(g^{\frac{1}{2}}\right)^{T}\left(R^{\frac{1}{2}}|x|\right)\right]^{2}=\left\|g^{\frac{1}{2}}\right\|^{2}\left\|R^{\frac{1}{2}} \mid x\right\|^{2} \quad \text { and } \quad e^{T} g=\omega .
$$

Since the vectors $g^{\frac{1}{2}}$ and $R^{\frac{1}{2}}|x|$ are nonzero we deduce from the first equality that $g_{v w}>0$ implies $x_{v w}>0$ and, moreover, for all such $\operatorname{arcs}(v, w)$ the products $r_{v w}\left|x_{v w}\right|$ have the same value. Since $r_{v w}\left|x_{v w}\right|$ is equal to the voltage over $\operatorname{arc}(v, w)$ we conclude that $g$ is optimal if and only if

$$
e^{T} g=\omega \text {, if } g_{v w}>0 \text { then } x_{v w} \neq 0 \text { and }\left|y_{v}-y_{w}\right| \text { is constant. }
$$

Using this one easily verifies that the second network in Figure 3 is optimal.

\subsubsection{Stability with respect to small perturbations}

In this section we consider an optimal network $g$ for a given external current $f$. One may wonder how the network behaves when it is fed with a current that differs slightly from the current $f$ for which it is designed. We will demonstrate in this section that very small perturbations in the design current $f$ may have a disastrous effect on the dissipation.

To make this clear, let $z$ be a nonzero eigenvector of $A(g)$ with eigenvalue $\lambda>0$. So we have $A(g) z=\lambda z \neq 0$. Let $f$ denote the design current of the network and $y$ the corresponding potential vector. Without loss of generality we assume that $\|z\|=\|f\|$ and $z^{T} f \geq 0$. Now consider the situation that the design current is replaced by

$$
f(\gamma):=f+\gamma z
$$

for some $\gamma \geq 0$. Then the potential vector $y(\gamma)$ under the new current follows by solving the equation $A(g) y(\gamma)=f+\gamma z$. Since $A(g) y=f$ and $A(g) z=\lambda z$, this gives

$$
y(\gamma)=y+\frac{\gamma}{\lambda} z
$$

The new dissipation then satisfies

$$
\operatorname{Diss}_{f(\gamma)}(g)=(f+\gamma z)^{T}\left(y+\frac{\gamma}{\lambda} z\right)=f^{T} y+\gamma z^{T} y+\frac{\gamma}{\lambda} f^{T} z+\frac{\gamma^{2}}{\lambda} z^{T} z .
$$

One has $f^{T} y=\operatorname{Diss}_{f}(g), f^{T} z \geq 0, z^{T} z=\|f\|^{2}$ and

$$
\lambda z^{T} y=y^{T}(\lambda z)=y^{T} A(g) z=z^{T}(A(g) y)=z^{T} f \geq 0,
$$

whence $z^{T} y \geq 0$. Thus we obtain 


\section{ROOS, Y. BAI and D. CHAERANI}

$$
\operatorname{Diss}_{f(\gamma)}(g) \geq \operatorname{Diss}_{f}(g)+\frac{\gamma^{2}}{\lambda}\|f\|^{2} .
$$

It thus follows that the effect on the dissipation of a small perturbation of the design current may be large if the eigenvalue $\lambda$ is small.

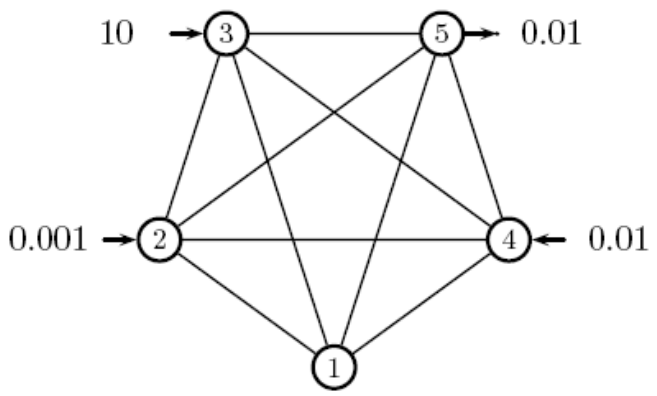

Figure 4. A 5-node network with given external currents in the free nodes.

\section{Example 3.4}

By way of example we consider the 5-node grid shown in Figure 4 with $\omega=10$, The nodes are numbered as indicated. Node 1 is fixed and the values of the input currents in the remaining nodes are as shown. The matrix $B$ and the input vector, which we denote as $f_{1}$, are as follows.

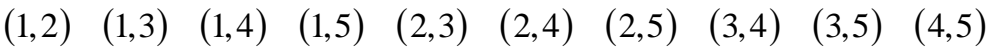

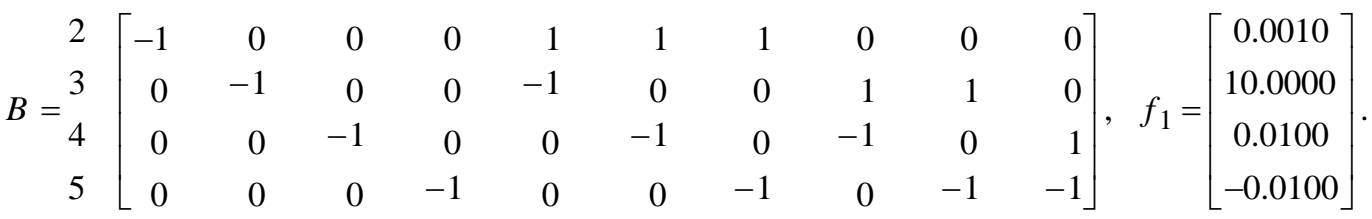

For the optimal solution of (14) the following holds:

$$
g_{1}:=g=\left[\begin{array}{l}
0.000571 \\
9.981628 \\
0.007812 \\
0.000000 \\
0.000000 \\
0.000000 \\
0.000428 \\
0.000000 \\
0.007384 \\
0.002177
\end{array}\right], \quad x=\left[\begin{array}{r}
-0.000572 \\
-9.992608 \\
-0.007820 \\
-0.000000 \\
0.000000 \\
0.000000 \\
0.000428 \\
0.000000 \\
0.007392 \\
0.002180
\end{array}\right], y=\left[\begin{array}{l}
1.001100 \\
1.001100 \\
1.001100 \\
0.000000
\end{array}\right] .
$$

Moreover, the dissipation equals 10.022012 . The matrix $A(g)$ is given by 


\section{ROBUST RESISTANCE NETWORK TOPOLOGY DESIGN}

$$
A(g)=B \operatorname{diag}(g) B^{T}=\left[\begin{array}{rrrr}
0.000999 & -0.000000 & -0.000000 & -0.000428 \\
-0.000000 & 9.989012 & -0.000000 & -0.007384 \\
-0.000000 & -0.000000 & 0.009989 & -0.002177 \\
-0.000428 & -0.007384 & -0.002177 & 0.009989
\end{array}\right],
$$

whose eigenvalues are $0.000977,0.007822,0.012172$, and 9.989018. So the smallest eigenvalue is $\lambda=0.000977$, and a corresponding eigenvector $u$ such that $\|u\|=\left\|f_{1}\right\|$ and $f_{1}^{T} u \geq 0$ is given by

$$
u=\left[\begin{array}{l}
9.986587 \\
0.000372 \\
0.121645 \\
0.503471
\end{array}\right] \text {. }
$$

Using (26) we derive that

$$
\operatorname{Diss}_{f_{1}(\gamma)}(g) \geq \operatorname{Diss}_{f_{1}}(g)+\frac{\gamma^{2}}{\lambda}\left\|f_{1}\right\|^{2} \geq \operatorname{Diss}_{f_{1}}(g)+102317 \gamma^{2} .
$$

Hence, for $\gamma=0.1$, the dissipation becomes about 1023. Thus we conclude that a perturbation of the design current of only $10 \%$ may lead to an increase of the dissipation by as much as a factor 100 . For future reference we give the perturbed external current yielding this increase in the dissipation:

$$
f_{2}:=f_{1}(0.1)=f_{1}+0.1 u=\left[\begin{array}{r}
0.999659 \\
10.000037 \\
0.022165 \\
0.040347
\end{array}\right] \text {. }
$$

The actual dissipation for $f_{2}$ is given by 1035.225318 .

The above example makes clear that a small perturbation of the design current may cause a large increase of the dissipation. In practice, this phenomenon may cause the network to be burned. So it is a an important question if we can find a network that is more robust with respect to small perturbations of the design current.

\subsection{Robust models for the RNTD problem}

In this section we start by showing that the RNTD problem can also be modelled as a semidefinite optimization (SDO) problem. An advantage of the SDO model is that it naturally extends to the multi-current case, i.e., the case where we want to design a network that is able to withstand a finite set of different currents in the best possible way. This enables us to improve the robustness of a network considerably.

\subsubsection{Semidefinite model for the RNTD problem}

It has become clear in the previous section that for a given network $g$ with external current $f$ the dissipation equals $f^{T} y$, where $y$ follows from the equation $A(g) y=f$. Hence we have

$$
\operatorname{Diss}_{f}(g)=f^{T} y=f^{T} A(g)^{-1} f .
$$

Theorem 3.5 $\operatorname{Diss}_{f}(g) \leq \tau$ holds if and only if

$$
\left[\begin{array}{cc}
\tau & f^{T} \\
f & A(g)
\end{array}\right] \succeq 0 .
$$

Proof. By (29), Diss $f(g) \leq \tau$ is equivalent to

$$
\tau-f^{T} A(g)^{-1} f \geq 0 .
$$




\section{ROOS, Y. BAI and D. CHAERANI}

By Lemma 5.1 this is equivalent to (30), proving the theorem.

Recall that the matrix $A(g)=B G B^{T}$ is linear in $G$. This means that (30) is an LMI. Due the semidefinite representation of the dissipation, as given by Theorem 3.5, we can formulate the RNTD problem as the following semidefinite optimization problem:

$$
\min _{\tau, g}\left\{\tau:\left[\begin{array}{cc}
\tau & f^{T} \\
f & A(g)
\end{array}\right] \succeq 0, e^{T} g \leq \omega, g \geq 0\right\} .
$$

\subsubsection{Semidefinite model for the multi-current RNTD problem}

A big advantage of the above model is that it can be easily adapted to obtain a RNTD that can best withstand a finite number of currents $f_{j}, 1 \leq j \leq k$ (not acting at the same time). We simply need to solve the following semidefinite problem:

$$
\min _{\tau, g}\left\{\tau:\left[\begin{array}{cc}
\tau & f_{j}^{T} \\
f_{j} & A(g)
\end{array}\right] \succeq 0, j=1, \cdots, k, e^{T} g \leq \omega, g \geq 0\right\} .
$$

The design variables are the components of the conductance vector $g$ and $\tau \in \mathbf{R}$. A crucial issue is that the matrices in (32) depend linearly on these variables. The corresponding inequalities in (32) are therefore called linear matrix inequalities (LMI's). They express that the highest dissipation, over the currents $f_{1}, \cdots, f_{k}$, of the network determined by $g$ does not exceed $\tau$, while the linear inequalities $e^{T} g \leq \omega$ and $g \geq 0$ express that $g=\left(g_{1}, \cdots, g_{n}\right)$ is an admissible network.

A natural question is whether we can solve this model efficiently. The answer is affirmative, as may be clear from Section 1 . So we are ready to give an example.

\section{Example 3.6}

We turn back to the problem considered in Example 3.4. There we considered a network for the single design current $f_{1}$, and we also subjected the resulting network to the current $f_{2}$, as given by (28). These forces are given by

$$
f_{1}=\left[\begin{array}{r}
0.0010 \\
10.0000 \\
0.0100 \\
-0.0100
\end{array}\right], \quad f_{2}=\left[\begin{array}{r}
0.999659 \\
10.000037 \\
0.022165 \\
0.040347
\end{array}\right] \text {. }
$$

Our aim is to optimize the network with respect to both currents by solving (32) for $k=2$ and $f_{1}$ and $f_{2}$, as just given. The optimal solution turns out to be

$$
g_{2}=\left[\begin{array}{l}
0.903687 \\
9.039812 \\
0.020035 \\
0.036466 \\
0.000000 \\
0.000000 \\
0.000000 \\
0.000000 \\
0.000000 \\
0.000000
\end{array}\right], \quad \tau_{2}=12.237244 .
$$




\section{ROBUST RESISTANCE NETWORK TOPOLOGY DESIGN}

The resulting network has a topology different from the network in Example 3.4, where $f_{1}$ was the design current. The dissipation of the new network with respect to $f_{1}$ is 11.069912. In Example 3.4 the dissipation was 10.022012. So the dissipation in the new network, when loaded with $f_{1}$, is about $10 \%$ higher than the minimal dissipation. This is the price we pay for more robustness, as becomes clear by computing the dissipation with respect to $f_{2}$, which is now 12.237244 , an increase of only $22 \%$. This is an enormous improvement with respect to the network of Example 3.4 where $f_{2}$ gave rise to an increase of the dissipation with a factor of more than 100.

Note that this result does not necessarily imply that the new network is stable with respect to other small perturbations of the current. We investigate the instability using the same approach as in Section 3.3.2. For the new network the smallest eigenvalue of $A(g)$ is $\lambda=0.020035$ and the corresponding (normalized) eigenvector $u$ such that $\|u\|=\left\|f_{1}\right\|$ and $f_{1}^{T} u \geq 0$ is given by

$$
u=\left[\begin{array}{r}
0.000000 \\
0.000000 \\
10.000010 \\
0.000001
\end{array}\right]
$$

If we replace the design current by $f_{1}(\gamma)=f_{1}+\gamma u$, with $\gamma=0.1$, we get

$$
f_{3}:=f_{1}(0.1)=f_{1}+0.1 u=\left[\begin{array}{r}
0.001000 \\
10.000000 \\
1.010001 \\
-0.010000
\end{array}\right] \text {. }
$$

The dissipation in the network then becomes 61.981951, an increase with more than a factor of 6 with respect to the minimal dissipation for $f_{1}$. Of course, we then can solve the multi-current model (32) for the input currents $f_{1}, f_{2}$, and $f_{3}$, which gives

$$
g_{3}:=\left[\begin{array}{r}
0.532924 \\
8.769407 \\
0.496593 \\
0.016689 \\
-0.000000 \\
0.170698 \\
-0.000000 \\
-0.000000 \\
-0.000000 \\
0.013690
\end{array}\right], \quad \tau_{3}:=13.003520
$$

The dissipations with respect to the respective input currents are now 11.406625, 13.003521 and 13.003521, which is a significant improvement. Note that the last two numbers are (a little) larger than $\tau_{3}$; this must be due to inaccuracy in the computations. For future use, we computed also a worst-case input current for this network, in the same way as before, which gives 


\section{ROOS, Y. BAI and D. CHAERANI}

$$
f_{4}=\left[\begin{array}{r}
-0.004708 \\
10.000000 \\
-0.012523 \\
-1.009731
\end{array}\right]
$$

It still may happen that some unforseen small perturbation of one of the three input currents $f_{i}$ in the last example may drastically increase the dissipation. In the next section we present a model which yields a network that withstands every perturbation of the design vector $f_{1}$, provided that the norm of the perturbation does not exceed a prescribed fraction of $\left\|f_{1}\right\|$.

\subsubsection{A more generic RNTD model}

When dealing with robust optimization models for the RTND problem the most natural situation to consider is the case where the input current may be modified by arbitrary small perturbations. We therefore consider the situation where the set of external currents $F$ has the form

$$
F=\left\{f=f^{n}+Q z: z^{T} z \leq 1\right\}, \quad Q \in \mathbf{M}^{m \times p},
$$

where $f^{n}$ is the design current and the ellipsoid $\left\{Q z: z^{T} z \leq 1\right\}$ contains all perturbations that might occur. The matrix $Q$ has to be chosen such that $F$ contains all possible external currents that the network has to withstand. Since the set $F$ is infinite, we meet a difficulty not present in the case of finite $F$, namely that the objective now is to minimize

$$
\operatorname{Diss}_{F}(g):=\sup _{f \in F} \operatorname{Diss}_{f}(g),
$$

which is the supremum of infinitely many semidefinite representable (SDR) functions. Fortunately, it is easy to get a semidefinite representation for $\operatorname{Diss}_{F}(g)$. In the next theorem $I_{p}$ denotes the unit matrix of order $p$.

Theorem 3.7 Let $F$ be as given by (35). Then one has $\operatorname{Diss}_{f}(g) \leq \tau$ for each $f \in F$ if and only if

$$
\left[\begin{array}{ccc}
\tau-\lambda & \left(f^{n}\right)^{T} & 0 \\
f^{n} & A(g) & Q \\
0 & Q^{T} & \lambda I_{p}
\end{array}\right] \succeq 0
$$

Proof. Due to Theorem 3.5 we have $\operatorname{Diss}_{F}(g) \leq \tau$ if and only if

$$
\left[\begin{array}{cc}
\tau & f^{T} \\
f & A(g)
\end{array}\right] \succeq 0, \quad \forall f \in F,
$$

or, equivalently, if and only if

$$
\left[\begin{array}{cc}
\tau & \left(f^{n}+Q z\right)^{T} \\
f^{n}+Q z & A(g)
\end{array}\right] \succeq 0, \quad \forall\left(z: z^{T} z \leq 1\right) .
$$

We have the following chain of equivalent statements, each being equivalent to the above property:

$$
\alpha^{2} \tau+2 \alpha\left(f^{n}+Q z\right)^{T} \beta+\beta^{T} A(g) \beta \geq 0, \quad \forall \alpha \in \mathbf{R}, \forall \beta \in \mathbf{R}^{V}, \forall\left(z: z^{T} z \leq 1\right)
$$




\section{ROBUST RESISTANCE NETWORK TOPOLOGY DESIGN}

$$
\begin{gathered}
\Leftrightarrow \quad \alpha^{2} \tau+2 \alpha\left(f^{n}\right)^{T} \beta+\beta^{T} A(g) \beta+2 \alpha z^{T} Q^{T} \beta \geq 0, \quad \forall \alpha \in \mathbf{R}, \forall \beta \in \mathbf{R}^{V}, \forall\left(z: z^{T} z \leq 1\right) \\
\Leftrightarrow \quad \alpha^{2} \tau+2 \alpha\left(f^{n}\right)^{T} \beta+\beta^{T} A(g) \beta+2 \min _{z: z^{T} z \leq 1} \alpha z^{T} Q^{T} \beta \geq 0, \quad \forall \alpha \in \mathbf{R}, \forall \beta \in \mathbf{R}^{V} \\
\Leftrightarrow \alpha^{2} \tau+2 \alpha\left(f^{n}\right)^{T} \beta+\beta^{T} A(g) \beta-2|\alpha|\left\|Q^{T} \beta\right\| \geq 0, \quad \forall \alpha \in \mathbf{R}, \forall \beta \in \mathbf{R}^{V} \\
\Leftrightarrow \quad \alpha^{2} \tau+2 \alpha\left(f^{n}\right)^{T} \beta+\beta^{T} A(g) \beta+2 \eta^{T} Q^{T} \beta \geq 0, \quad \forall \alpha \in \mathbf{R}, \forall \beta \in \mathbf{R}^{V}, \forall\left(\eta: \eta^{T} \eta \leq \alpha^{2}\right) .
\end{gathered}
$$

Thus it becomes clear that $\operatorname{Diss}_{F}(g) \leq \tau$ holds if and only if, for all $\alpha, \beta, \eta$ :

$$
\eta^{T} \eta \leq \alpha^{2} \Rightarrow \alpha^{2} \tau+2 \alpha\left(f^{n}\right)^{T} \beta+\beta^{T} A(g) \beta+2 \eta^{T} Q^{T} \beta \geq 0 .
$$

By the $S$-lemma (Lemma 5.2) this holds if and only if there exists $\lambda \geq 0$ such that

$$
\alpha^{2} \tau+2 \alpha\left(f^{n}\right)^{T} \beta+\beta^{T} A(g) \beta+2 \eta^{T} Q^{T} \beta \geq \lambda\left(\alpha^{2}-\eta^{T} \eta\right)
$$

which can be written as

$$
\alpha^{2}(\tau-\lambda)+2 \alpha\left(f^{n}\right)^{T} \beta+\beta^{T} A(g) \beta+2 \eta^{T} Q^{T} \beta+\lambda \eta^{T} \eta \geq 0 .
$$

This implies the statement in the theorem.

An immediate consequence of the above theorem is that

$$
\operatorname{Diss}_{F}(g)=\min _{\tau, g}\left\{\tau:\left[\begin{array}{ccc}
\tau-\lambda & \left(f^{n}\right)^{T} & 0 \\
f^{n} & A(g) & Q \\
0 & Q^{T} & \lambda I
\end{array}\right] \succeq 0, \quad e^{T} g \leq \omega, \quad g \geq 0\right\} .
$$

\section{Example 3.8}

In order to get a 5-node network with design current $f_{1}$, and $\omega=10$, which is robust with respect to arbitrary perturbations $u$, with $\|u\| \leq 0.1\left\|f_{1}\right\|$ we solve (32) with $f^{n}=f_{1}$ and $Q$ the identity matrix. This gives $\tau_{4}=14.414417$ and

$$
g_{4}=\left[\begin{array}{l}
0.417292 \\
8.741458 \\
0.424783 \\
0.408135 \\
0.000000 \\
0.000000 \\
0.000005 \\
0.000000 \\
0.008310 \\
0.000019
\end{array}\right], \quad \tau_{4}=14.414417
$$




\section{ROOS, Y. BAI and D. CHAERANI}

This network admits perturbations up to $10 \%$ of the design current, while the dissipation will never exceed 14.414417. The dissipations for $f_{1}, f_{2}, f_{3}$ and $f_{4}$ are 11.429114, 13.830830, 13.830830, and 13.831614, respectively. These values are all well below $\tau_{4}$.

Table 3. Optimal networks and their dissipations

\begin{tabular}{|l|ccccc|}
\hline 0 & $g$ & $g_{1}$ & $g_{2}$ & $g_{3}$ & $g_{4}$ \\
\hline 1 & $g_{12}$ & 0.000571 & 0.903687 & 0.532924 & 0.417292 \\
2 & $g_{13}$ & 9.981628 & 9.039812 & 8.769407 & 8.741458 \\
3 & $g_{14}$ & 0.007812 & 0.020035 & 0.496593 & 0.424783 \\
4 & $g_{15}$ & 0.000000 & 0.036466 & 0.016689 & 0.408135 \\
5 & $g_{23}$ & 0.000000 & 0.000000 & 0.000000 & 0.000000 \\
6 & $g_{24}$ & 0.000000 & 0.000000 & 0.170698 & 0.000000 \\
7 & $g_{25}$ & 0.000428 & 0.000000 & 0.000000 & 0.000005 \\
8 & $g_{34}$ & 0.000000 & 0.000000 & 0.000000 & 0.000000 \\
9 & $g_{35}$ & 0.007384 & 0.000000 & 0.000000 & 0.008310 \\
10 & $g_{45}$ & 0.002177 & 0.000000 & 0.013690 & 0.000019 \\
11 & design dissipation $\tau$ & 10.022012 & 12.237244 & 13.003520 & 14.414417 \\
12 & dissipation w.r.t $f_{1}$ & 10.022012 & 11.069912 & 11.406625 & 11.429114 \\
13 & dissipation w.r.t $f_{2}$ & 1035.225318 & 12.237244 & 13.003521 & 13.830830 \\
14 & dissipation w.r.t $f_{3}$ & 117.229046 & 61.981951 & 13.003520 & 13.830238 \\
15 & dissipation w.r.t $f_{4}$ & 118.800746 & 39.028988 & 45.311988 & 13.831614 \\
16 & $\lambda_{\text {min }}(A(g))$ & 0.000977 & 0.020035 & 0.030070 & 0.416460 \\
\hline
\end{tabular}

\subsubsection{Summary of the results}

Let us summarize the results of this section. In Section 3.2 we started by presenting the basic model (14) for the RNTD problem. This model contains the bilinear equality constraint $A(g) y=f_{1}$, which makes it hard to solve. Surprisingly enough it became clear in Section 3.3 that the solution of (14) can be constructed from the solution of the linear optimization problem (15) and its dual problem (16). The bad news of Section 3.3.2 is that the network thus obtained may suffer from serious instability. We showed in Example 3.4 that a $10 \%$ perturbation in the design current $f_{1}$ may multiply the dissipation by a factor of 1000 .

The good news is in Section 3.4. We first presented the multi-current model (32) in Section 3.4.2, which already gave a substantially improved robustness. Next, in Section 3.4.3 we derived the model (37), which yields a network that is stable with arbitrary perturbations of prescribed size. We demonstrated this in some examples. Table 3 summarizes the results of the examples in Section 3.4.

The last four columns in Table 3 correspond to the networks $g_{i}, 1 \leq i \leq 4$, that we found in these examples. The zero entries in $g_{i}$ refer to edges that are not used. Note that arcs $(2,3)$ and $(3,4)$ are never used. The table shows the dissipations for these networks for the input currents $f_{1}, f_{2}, f_{3}$ and $f_{4}$. One has $\left\|f_{1}-f_{i}\right\|=0.1\left\|f_{1}\right\|$ for $i \geq 3$. In other words, each $f_{i}$ with $i \geq 3$ is a $10 \%$ perturbation of $f_{1}$. Recall that $g_{1}$ is optimal for $f_{1}$ and $g_{2}$ is optimal for $\left\{f_{1}, f_{2}\right\}$. The network $g_{3}$ is optimal for $\left\{f_{1}, f_{2}, f_{3}\right\}$, and $g_{4}$ is such that for all $10 \%$ perturbations of $f_{1}$ the dissipation will be at most $\tau_{4}=14.414417$. The last row gives the smallest 


\section{ROBUST RESISTANCE NETWORK TOPOLOGY DESIGN}

eigenvalue of the conductance matrices $A\left(g_{i}\right)$. We saw in Section 3.3.2 that this eigenvalue can be considered as a measure for the robustness of a network. Indeed, the last row shows that this eigenvalue is increasing in $i$.

\section{Concluding remarks}

It is now well-known that $\mathrm{CO}$ is a powerful tool for the mathematical modelling of inherently nonlinear problems. The subject thanks its existence to the development of efficient solution methods for $\mathrm{CO}$ problems in the last decade. In particular, the possibility of modeling robustness of a design in a computationally tractable way opens up many new applications. A major source for the techniques used in this paper is Ben-Tal and Nemirovski (2001), where one of the striking examples concerns the development of CO models for robust truss topology design. In the present paper we apply the same approach to the inherently more simple case of the robust resistor network topology design problem. It is shown that, by using semidefinite models, the robustness of the design can be significantly improved. The extension to more general electrical networks, with inductances and capacitors, seems to be a promising subject for future research.

\section{Appendix}

To make the paper self-supporting we list in this appendix two lemmas that are used in the paper. For the proofs we refer to the literature. The first lemma is known as the Schur complement lemma.

Lemma 5.1 (Lemma 4.2.1, page 148 in (Ben-Tal and Nemirovski, 2001))

Let $A=\left[\begin{array}{cc}B & C^{T} \\ C & D\end{array}\right]$ be a symmetric matrix with $k \times k$ block $B$ and $\ell \times \ell$ block $D$. Assume that $B$ is positive definite. Then $D-C B^{-1} C^{T}$ is called the Schur complement of $B$ in $A$. One has

$$
A \succeq 0 \Leftrightarrow D-C B^{-1} C^{T} \succeq 0 .
$$

We conclude with the so-called $S$-lemma.

Lemma 5.2 (Theorem 4.3.3, page 176 in Ben-Tal and Nemirovski (2001))

Let $A$ and $B$ be symmetric $n \times n$ matrices, and assume that $x^{T} A x>0$ holds for some $x$. Then

$$
x^{T} A x \geq 0 \Rightarrow x^{T} B x \geq 0
$$

holds for all $x$ if and only if there exists a $\lambda \geq 0$ such that $B \succeq \lambda A$.

\section{References}

AITCHISON, R.E. 1964. Resistance between adjacent points of Liebman mesh. Am. J. Phys., 32: 566-566.

BELEVITCH, V. 1968. Classical Network Theory. Holden-Day, San Francisco, California, USA.

BEN-TAL, A., EL GHAOUI, L. and NEMIROVSKI, A. 2000. Robustness. In Handbook of Semidefinite Programming, Internat. Ser. Oper. Res. Management Sci., 27: 139-162.

BEN-TAL, A., EL GHAOUI, L. and NEMIROVSKI, A. 2009. Robust Optimization. Princeton Series in Applied Optimization. Princeton University Press, Princeton, USA.

BEN-TAL, A., MARGALIT, T. AND NEMIROVSKI, A. 1999. Robust modeling of multi-stage portfolio problems. In S. ZHANG, H. FRENK, C. ROOS, and T. TERLAKY (eds.), High Performence Optimization Techniques, pp. 303-328. Kluwer Academic Publishers, Dordrecht, The Netherlands.

BEN-TAL, A. and NEMIROVSKI, A. 1997. Robust truss topology design via semidefinite programming. SIAM Journal on Optimization, 7(4): 991-1016. 


\section{ROOS, Y. BAI and D. CHAERANI}

BEN-TAL, A. and NEMIROVSKI, A. 1998. Robust convex optimization. Math. Oper. Res., 23(4): $769-805$.

BEN-TAL, A. and NEMIROVSKI, A. 1999. Robust solutions of uncertain linear programs. Oper. Res. Lett., 25(1): 1-13.

BEN-TAL, A. and NEMIROVSKI, A. 2000. Robust solutions of linear programming problems contaminated with uncertain data. Mathematical Programming, 88(3, Ser. A): 411-424.

BEN-TAL, A. and NEMIROVSKI, A. 2001. Lectures on Modern Convex Optimization. Analysis, Algorithms and Engineering Applications, volume 2 of MPS-SIAM Series on Optimization. SIAM, Philadelphia, USA.

BEN-TAL, A. and NEMIROVSKI, A. 2002. On tractable approximations of uncertain linear matrix inequalities affected by interval uncertainty. SIAM Journal on Optimization, 12(3): 811-833 (electronic).

BEN-TAL, A. and NEMIROVSKI, A. 2002. Robust optimization-methodology and applications. Mathematical Programming, 92(3, Ser. B): 453-480.

EL GHAOUI, L. AND LEBRET, H. 1997. Robust solutions to least-squares problems with uncertain data. SIAM Journal on Matrix Analysis and Applications, 18(4): 1035-1064.

EL GHAOUI, L., OUSTRY, F. and LEBRET, H. 1999. Robust solutions to uncertain semidefinite programs. SIAM Journal on Optimization, 9(1): 33-52 (electronic).

GHOSH, A., BOYD, S. and SABERI, A. 2008. Minimizing effective resistance of a graph. SIAM Review, 50: 37-66.

NESTEROV, Y. and NEMIROVSKI, A. 1994. Interior-Point Polynomial Algorithms in Convex Programming, volume 13 of SIAM Studies in Applied Mathematics. SIAM, Philadelphia, USA.

ROOS, C., TERLAKY, T. and VIAL, J.-PH. 2005. Theory and Algorithms for Linear Optimization. Springer, Chichester, UK (1st Edition, Theory and Algorithms for Linear Optimization. An Interior-Point Approach. John Wiley \& Sons, 1997).

SOYSTER, A.L. 1973. Convex programming with set-inclusive constraints and applications to inexact linear programming. Operations Research, 21(5): 1154-1157.

TERLAKY, T. (Ed.) 1996. Interior Point Methods of Mathematical Programming, volume 5 of Applied Optimization. Kluwer Academic Publishers, Dordrecht, The Netherlands.

WRIGHT, S.J. 1997. Primal-Dual Interior-Point Methods. SIAM, Philadelphia, USA.

YE, Y. 1997. Interior Point Algorithms. Wiley-Interscience Series in Discrete Mathematics and Optimization. John Wiley \& Sons Inc., New York, USA.

Received 11 April 2011

Accepted 12 September 2011 